Reprod. Nutr. Dévelop., 1984, 24 (2), 117-125.

\title{
Absence de transfert intestinal de protéines chez la truite arc-en-ciel, Salmo gairdnerii, Rich.
}

\author{
G. MARCOTTE, J. DE LA NOÜE
}

Département de Biologie et Centre de Recherche en Nutrition

Université Laval, Québec GIK 7P4, Canada.

Summary. Absence of intestinal transfer of proteins in the rainbow trout, Salmo gairdnerii, Rich.

Previous electron microscopic observations made by some authors have led to the hypothesis that intact protein molecules might be absorbed by the hind-gut of rainbow trout (Salmo gairdneri, Rich.). This paper reports a study of serosal transfer of $\alpha_{\mathrm{s}}$-casein using everted intestinal sacs. The results show that, although protein molecules of endogenous origin appeared on the serosal side, there was no detectable serosal transfer of the $\alpha_{\mathrm{s}}$-casein initially present in the mucosal compartment. Protein hydrolysis occurred in the mucosal compartment, whereas nothing similar was observed on the serosal side. Our results indicate that if there was some uptake of proteins by posterior intestine, it would be followed by intracellular hydrolysis.

\section{Introduction.}

II est bien connu que l'intestin grêle de certains mammifères nouveau-nés transfère des macromolécules, ce qui permet l'acquisition de l'immunité passive (Morris, 1968). De nombreuses études de ce type d'absorption ont été réalisées chez différentes espèces animales, notamment chez le Rat (Clark, 1959 ; Jones, 1976 ; Williams et Hemmings, 1978), la Souris (Hugon, 1971), et le Porc (Brambell, 1958 ; Kraehenbuhl et Campiche, 1969). Ces différents auteurs admettent que l'absorption de ces substances se réalise par pinocytose. Les vésicules formées par les invaginations de la membrane plasmique entre les microvillosités se fusionnent pour former les vacuoles. Celles-ci, contenant les anticorps, sont transférées vers la surface sérosale sans interférence des autres organites cellulaires ou se fusionnent dans la région supranucléaire où les lysosomes peuvent les dégrader (Kraehenbuhl et Campiche, 1969 ; Rodewald, 1973). De plus, le transfert intestinal de protéine exogène, pour des fins nutritionnelles, a été retenu chez la Carpe adulte (Noalliac-Depeyre et Gas, 1973) et la Truite adulte (Ezeasor et Stokoe, 1981).

Si I'on prend en considération 1) que la Truite arc-en-ciel possède un intestin court, caractéristique des Téléostéens carnivores, et que son rapport longueur du 
corps sur longueur de l'intestin est d'environ $5: 6$ (Ezeasor et Stokoe, 1980) ; 2) que les besoins en protéines pour une croissance optimale semblent élevés chez les Salmonidés comparativement à ceux des Vertébrés supérieurs (Luquet et Kaushik, 1979) ; 3) que la membrane plasmique des entérocytes du segment intestinal postérieur de la Truite arc-en-ciel, Salmo gairdnerii, Rich. montre des invaginations, signe avant-coureur de la pinocytose (Bergot, 1976 ; Iwai, 1968) ; 4) que la vaccination par voie orale présente un intérêt potentiel indéniable chez la Truite, nous avons estimé opportun de chercher à quantifier le transfert intestinal de protéines intactes chez cette espèce.

\section{Matériel et méthodes.}

Maintien des poissons. - Des truites arc-en-ciel (Salmo gairdnerii, Rich.) de $23,0 \pm 2,5 \mathrm{~cm}(163,2 \pm 54,0 \mathrm{~g})$, obtenues d'une pisciculture locale (Aulnaiesous-Bois, St-Féréol, Québec), sont maintenues en aquarium à raison d'un animal par 30 litres d'eau. Une photopériode (lumière : obscurité, $12: 12$ ) est établie et la température est maintenue à $12 \pm 1{ }^{\circ} \mathrm{C}$. Un filtre bactérien nitrifiant (Liao et Mayo, 1972 ; Pettigrew et al., 1978) reconditionne l'eau du robinet préalablement déchlorée par aération. Un renouvellement de l'eau $(50 \%)$ est effectué tous les sept jours afin de diminuer la concentration des nitrates produits par le filtre bactérien. Les poissons sont nourris quotidiennement de granulés commerciaux (firme Ewos) d'un diamètre de 3,2 mm. Le jour de l'expérience, les animaux faisant l'objet d'étude ne reçoivent pas leur ration quotidienne.

Montage intestinal. - Après sacrifice de l'animal par traumatisme crânien, l'intestin postérieur ou rectum (Bertin, 1958 ; Burnstock, 1959) est tout d'abord libéré des vaisseaux sanguins et des réserves lipidiques. Par la suite, le segment intestinal est incubé selon la méthode des sacs retournés (Wilson et Wiseman, 1954), I'une des extrémités étant montée sur une canule (Crane et Wilson, 1958). Les incubations sont réalisées par paires, à une température de $12 \pm 0,1^{\circ} \mathrm{C}$, l'une des incubations étant conduite en présence de glucose $(20 \mathrm{mM})$, l'autre sans glucose. La composition de la solution physiologique (Holmes et Scott, 1960) apparaît au tableau 1.

TABLEAU 1

Composition de la solution physiologique $(\mathrm{pH} \mathrm{7,4)}$.

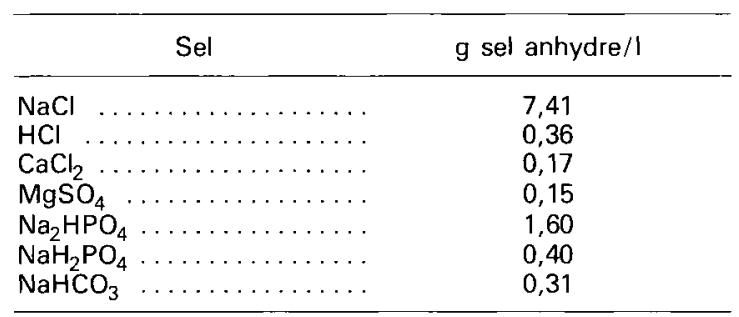


Nous avons isolé la fraction $\alpha_{\mathrm{s}}$ à partir de la micelle de caséine du lait (Sigma Chemical Company, St-Louis, Mo) selon la méthode de Tripathi et Gehrke (1970). Une dialyse avec le tampon A (tabl. 2) nous permet de solubiliser la fraction $\alpha_{\mathrm{s}}$, conservée par la suite lyophilisée. Nous avons également utilisé de l' $\alpha_{s}$-caséine commerciale (Sigma Chem. Co., St-Louis, Mo), de même comportement électrophorétique.

Dans la solution mucosale, la concentration initiale de la caséine $\alpha_{\mathrm{s}}$ est de $1 \%(P / V)$ pour des incubations de $30 \mathrm{~min}(\mathrm{n}=3$ ) et $60 \mathrm{~min}(\mathrm{n}=6)$. Lors des incubations à une concentration mucosale protéique initiale de $3 \%$ ( $P / V)$, la durée d'incubation est de $30 \mathrm{~min}(n=2)$. Un barbotage continu d'air dans la solution mucosale assure un mélange et un apport en oxygène adéquats.

Les échantillons de la solution mucosale prélevés durant et après l'incubation sont mélangés avec le tampon B (tabl. 2) afin d'obtenir une concentration protéique maximale de $1 \mathrm{mg} / \mathrm{ml}$, cette concentration permettant une bonne séparation électrophorétique. A la fin de la période d'incubation, la face mucosale du sac intestinal est soigneusement rincée avec la solution physiologique afin d'éviter une contamination ultérieure de la solution sérosale, puis épongée. Une incision est effectuée à l'extrémité libre du sac intestinal et la solution sérosale est récupérée pour être ensuite ajustée avec le tampon $B$ jusqu'à un volume final de 0,2 ou $0,3 \mathrm{ml}$. A la suite du mélange avec le tampon $B$, tous les échantillons sont chauffés à $100^{\circ} \mathrm{C}$ (bain-marie) pendant $5 \mathrm{~min}$ afin d'éviter une hydrolyse subséquente à l'incubation. Un volume équivalent d'urée $4 \mathrm{M}$ est alors mélangé à l'échantillon refroidi, puis une goưtte de bromophénol bleu (saturé dans l'acide acétique $7 \%$ (V/V) complète la préparation des échantillons.

Electrophorèse du gel. - Les protéines des divers échantillons sont déterminées par électrophorèse sur gel de polyacrylamide $7 \%(P / V)$ en présence d'urée selon la méthode de Jovin et al. (1964), telle que décrite par Simard et al. (1979). La composition du gel de séparation est décrite au tableau 3. La polymérisation s'effectue dans des tubes transparents de $5 \mathrm{~mm}$ de diamètre intérieur, d'une longueur de $75 \mathrm{~mm}$. Les tubes sont placés dans une cellule d'électrophorèse (Bio

TABLEAU 2

Composition des tampons.

\begin{tabular}{clr}
\hline Tampon & Réactifs $/ 1000 \mathrm{ml}\left(\mathrm{H}_{2} \mathrm{O}\right)$ \\
\hline «A » & Tris (a) & $5,21 \mathrm{~g}$ \\
Dialyse & Glycine & $34,53 \mathrm{~g}$ \\
$\mathrm{pH} \mathrm{8,2}$ & EDTA NA $_{2}$ & $0,64 \mathrm{~g}$ \\
«B » & $\mathrm{H}_{3} \mathrm{PO}_{4} 1 \mathrm{M}$ & $128,0 \mathrm{ml}$ \\
Échantillon & Tris & $28,5 \mathrm{~g}$ \\
$\mathrm{pH} \mathrm{7,8}$ & Urée 10 M & $800,0 \mathrm{ml}$ \\
« C » & Glycine & $144,0 \mathrm{~g}$ \\
Ėlectrodes & Tris & $30,0 \mathrm{~g}$ \\
$\mathrm{pH} 8,3$ & à diluer $1: 9$ & \\
\hline
\end{tabular}

(a) (Hydro-méthyl) amino méthane.

(b) Acide éthylène diamine tétraacétique-disodique. 
TABLEAU 3

Composition du gel de séparation.

\begin{tabular}{lcl}
\multicolumn{2}{c}{ Réactifs $/ 100 \mathrm{ml}\left(\mathrm{H}_{2} \mathrm{O}\right)$} & Volumes $^{\text {(a) }}$ \\
\hline Acrylamide & $28,0 \mathrm{~g}$ & \\
Bis (b) & $0,735 \mathrm{~g}$ & 1 \\
Urée $10 \mathrm{M}$ & $80,0 \mathrm{ml}$ & \\
$\mathrm{HCl} 1 \mathrm{~N}$ & $12,0 \mathrm{~g}$ & \\
Tris pH 8,9 & $9,07 \mathrm{~g}$ & 2 \\
Temed (c) & $0,12 \mathrm{ml}$ & \\
Urée $10 \mathrm{M}$ & $80,0 \mathrm{ml}$ & \\
$\left(\mathrm{NH}_{4}\right)_{2} \mathrm{~S}_{2} \mathrm{O}_{8}$ & $0,28 \mathrm{~g}$ & 1 \\
Urée 10 M & $80,0 \mathrm{ml}$ & 1 \\
\hline
\end{tabular}

(a) Mélange des solutions mères; (b) $N, N^{\prime}$-Méthylène - Bis - Acrylamide ; (c) $N, N, N^{\prime}, N^{\prime}$ Tétraméthyl-Éthylènediamine.

Rad's, model 155) contenant le tampon C (tabl. 2). Les échantillons (50 $\mu \mathrm{L}$ ) sont déposés sur le gel et la migration s'effectue à une intensité de 4 milliampères par tube.

A la fin de la migration, le gel est récupéré et le front de migration est marqué. La coloration, d'une durée d'une heure, est réalisée au moyen du bleu de Coomassie G-250 (Reisner et al., 1975). Une fois la coloration stabilisée (24 h, acide acétique $7 \%(\mathrm{~V} / \mathrm{V})$, des densitogrammes des gels sont alors réalisés (Densicomp model 445, Electrophoresis densitometer, Clifford Instruments Inc., Natick, Ma). L'identification des bandes protéiques est faite par rapport au front de migration (Rf).

\section{Résultats.}

La méthode des sacs retournés nous a permis de déceler l'apparition de substances dans le compartiment sérosal ainsi que les modifications du contenu mucosal. Les échantillons de la solution mucosale ( $n=7)$ montrent une hydrolyse enzymatique de la caséine $\alpha_{\mathrm{s}}$ (fig. 1). Toutefois, on ne dénote aucun changement de solution mucosale durant quatre autres incubations supplémentées en D-glucose et trois autres incubations non supplémentées. De plus, le mélange d'une solution de caséine $\alpha_{\mathrm{s}} 1 \%$ (P/V) supplémentée en D-glucose avec la solution mucosale finale ( $t=60 \mathrm{~min}$ ) provenant d'une incubation réalisée sans protéine (fig. 1) montre une hydrolyse enzymatique.

Les densitogrammes de la solution sérosale des segments incubés en présence de caséine $\alpha_{\mathrm{s}}$ montrent l'apparition de bandes protéiques (fig. 2 et 3). La mobilité électrophorétique de la caséine $\alpha_{\mathrm{s}}(1,69)$ et celle de la fraction caséine $k$ $(1,47)$ servent de référence. Les bandes protéiques de la solution sérosale ont des rapports de migration de 1,$23 ; 1,40 ; 1,57$ ou 1,63 .

Des séparations électrophorétiques d'un intestin lyophilisé (non incubé) et consécutives à une incubation dans la solution physiologique sans protéine ont 


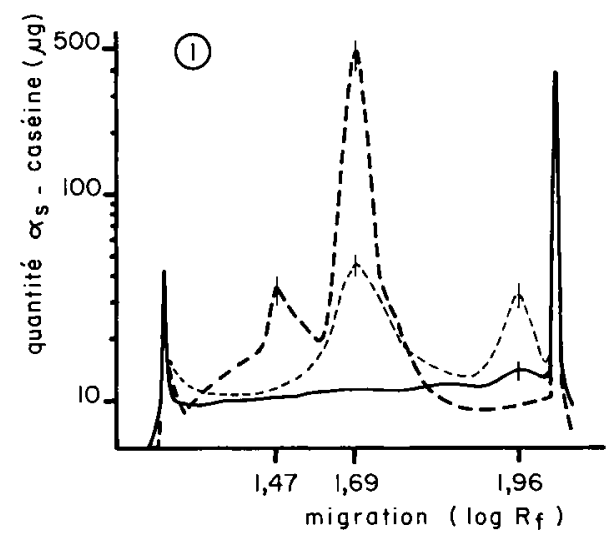

FIG. 1. - Modifications du contenu de la solution mucosale. Densitogrammes de gels obtenus: a) des solutions mucosales à $t_{0}(-\ldots)$ et à $t_{60}(\ldots, \ldots)$ de segments incubés en présence de la caséine $\alpha_{\mathrm{s}} 1 \%(\mathrm{P} / \mathrm{V})$ mucosale supplémentée en glucose $\left.(20 \mathrm{mM}) ; b\right)$ du mélange $(\mathrm{t}=5 \mathrm{~min}$, ) d'une solution identique de caséine $\alpha_{\mathrm{s}}$ avec la solution mucosale finale ( $t=60 \mathrm{~min}$ ) d'un segment incubé en présence de la solution physiologique sans protéine. Rf. 1,47 : caséine $k$; 1,69 : caséine $\alpha_{s} ; 1,96$ : produit d'hydrolyse.
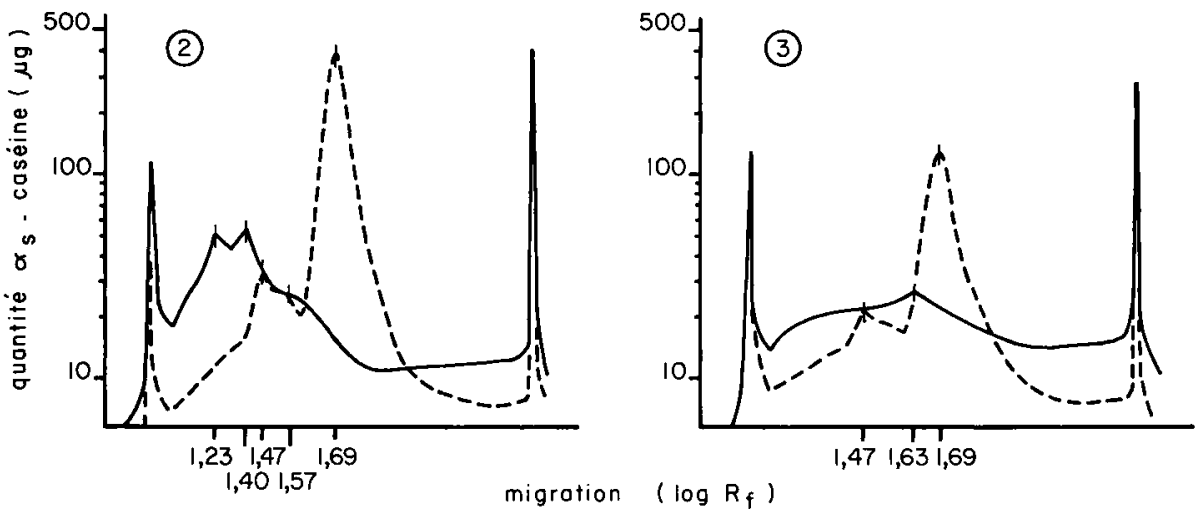

FIG. 2. - Apparition de protéines dans la solution sérosale. Densitogrammes de gels obtenus à partir de la solution sérosale finale $(t=60 \mathrm{~min})\left({ }_{(}-\right.$d'un segment incubé en présence d'une solution mucosale initiale $(---)$ de caséine $x_{s} 1 \%(P / V)$ supplémentée en glucose. Rf. 1,23: protéine endogène ; 1,40 : protéine endogène ; 1,57 : protéine endogène $; 1,47$ : caséine $\kappa ; 1,69$ : caséine $\alpha_{\mathrm{s}}$.

FIG. 3. - Apparition de protéines dans la solution sérosale. Densitogrammes de gels obtenus à partir de la solution sérosale (— finale $(t=30 \mathrm{~min})$ d'un segment incubé en présence d'une solution mucosale initiale $(\ldots)$ de caséine $\alpha_{s} 1 \%$ (P/V). Rf. 1,63 : protéine endogène ; 1,47 : caséine $\kappa ; 1,69$ : caséine $x_{\mathrm{s}}$. 
également été effectuées (fig. 4). Les bandes protéiques de la solution sérosale ont alors des rapports de migration de 1,$21 ; 1,38 ; 1,55 ; 1,63$ et 1,84 . Le petit nombre de bandes protéiques révélées pour l'intestin lyophilisé s'explique par une détection limitée par le type et la concentration du gel. Les caractéristiques du gel ont été choisies de façon à obtenir une migration suffisamment importante de la caséine $\alpha_{\mathrm{s}}$ de même qu'un empilement maximal (bande mince).

Les résultats présentés à la figure 5 prouvent qu'aucune hydrolyse de la caséine $\alpha_{\mathrm{s}}$ introduite dans le compartiment sérosal n'est détectable après une incubation de $30 \mathrm{~min}$ en présence de glucose $(20 \mathrm{mM})$. De plus, l'absence de glucose dans la solution mucosale mène à des résultats (non illustrés) identiques à ceux que l'on a obtenus en sa présence (fig. 5).
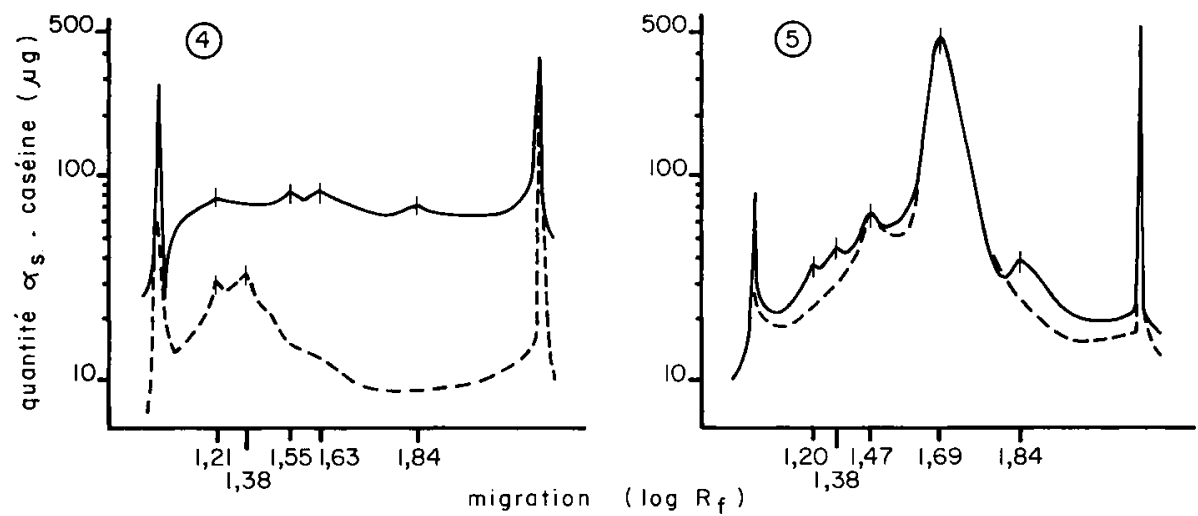

FIG. 4. - Apparition de protéines endogènes. Densitogrammes de gels obtenus : al d'un intestin lyophilisé non incubé (—) et $b$ ) de la solution sérosale $(-\cdots)$ finale $(t=60 \mathrm{~min}$ ) d'un segment incubé en présence de la solution physiologique sans protéine. Toutes les bandes identifiées correspondent à des protéines d'origine endogène.

FIG. 5. - Modifications du contenu de la solution sérosale. Densitogrammes de gels obtenus: a) pour une solution de caséine $\alpha_{s}$ introduite dans le compartiment sérosal $(---)$ et $b$ ) à la suite d'une incubation de $30 \mathrm{~min}(-)$ en présence de D-glucose $(20 \mathrm{mM})$. Rf. 1,20: protéine endogène: 1,38 : protéine endogène ; 1,84 : protéine endogène : 1,47 : caséine $k$; 1,69 : caséine $x_{\mathrm{s}}$.

\section{Discussion et conclusions.}

Dans la présente étude, nous observons une hydrolyse rapide de la caséine $\alpha_{s}$ mucosale fort probablement causée par une libération d'enzymes par l'intestin postérieur (fig. 1). Toutefois, les changements qui surviennent dans la solution mucosale semblent indépendants de la supplémentation en D-glucose $(20 \mathrm{mM})$. Lors d'une étude de l'absorption des acides aminés, Bogé et al. (1979) ont d'ailleurs démontré que l'apport énergétique nécessaire au transport actif des acides aminés est endogène, le glucose de la solution mucosale n'y jouant aucun rôle. 
Nous remarquons également que la solution sérosale ne contient jamais la fraction caséine $\alpha_{\mathrm{s}}$ présente initialement dans la solution mucosale. Sachant que, contrairement au gel en présence de SDS (dodecylsulfate de sodium), dont la migration est basée sur le poids moléculaire, l'utilisation d'un gel de polyacrylamide en présence d'urée permet une séparation électrophorétique selon la charge de la protéine, on pourrait penser que les bandes retrouvées dans la solution sérosale (fig. 2 et 3) sont des fragments de la caséine $\alpha_{s}$. Cependant, les résultats des incubations " témoins " (fig. 4) nous démontrent que ces bandes protéiques sont indubitablement de source endogène. II convient de préciser que la concentration protéique de $3 \%$ (P/V) présente initialement dans la solution mucosale $n^{\prime}$ a pas favorisé le transfert sérosal de la caséine $\alpha_{\mathrm{s}}$.

L'impossibilité de démontrer un transfert sérosal de la caséine $\alpha_{\mathrm{s}}$ pourrait s'expliquer par certaines caractéristiques de cette protéine, telles que la charge, la conformation, etc. Cette explication ne devrait cependant pas être retenue, car les essais d'incubation menés avec une autre protéine aux caractéristiques très différentes, l'immunoglobuline du colostrum de bœuf, ont donné des résultats tout à fait similaires à ceux obtenus en présence de la caséine $\alpha_{\mathrm{s}}$ (absence de transfert sérosal). De plus, l'absence de transfert sérosal de la caséine $\alpha_{\mathrm{s}}$ ne peut être imputable à la non-viabilité du montage expérimental (Marcotte et de la Noüe, 1983).

Selon Iwai (1968), le mode d'absorption pour les cellules épithéliales de l'intestin des alevins de Truite est virtuellement le même que pour d'autres Téléostéens, tel le Poisson rouge. Le phénomène de la pinocytose dans l'intestin postérieur devrait donc permettre l'absorption de protéines, tout comme dans l'intestin grêle de certains Mammifères nouveau-nés. Cependant, Yamamoto (1966) a démontré que l'intestin de la Truite adulte diffère de celui du Poisson rouge. On ne retrouve pas d'invagination entre les microvillosités des cellules épithéliales, signe habituel d'activité pinocytosique. Cette différence serait attribuable à la présence d'un estomac bien développé chez la Truite adulte. La capacité d'absorber des macromolécules protéiques par pinocytose serait donc temporaire, comme chez les Mammifères. Pourtant, les études au microscope électronique de Bergot (1976) et de Ezeasor et Stokoe (1981), sur les entérocytes de l'intestin postérieur de la Truite arc-en-ciel, ont démontré la présence d'invaginations et de vésicules tout à fait comparables à celles du Poisson rouge et de l'avelin de la Truite arc-enciel. Sur ces bases, l'absorption de protéines par pinocytose serait un phénomène durable pour la partie postérieure de l'intestin de Truite. Nos résultats ne vérifient cependant pas la possibilité du transfert sérosal de la caséine $\alpha_{\mathrm{s}}$ et de l'immunoglobine, et nous indiquent qu'à l'instar du Lapin (Kraehenbuhl et Campiche, 1969), la Truite arc-en-ciel ne transfère pas les protéines intactes. On peut supposer que, s'il y a prise en charge des protéines par micropinocytose, celle-ci est suivie d'une hydrolyse intracellulaire, mécanisme qui s'ajouterait à la digestion intraluminale, avec pour conséquence une absorption plus importante des protéines ingérées. Cette explication est d'autant plus plausible que les besoins en azote d'origine protéique sont très élevés chez la Truite (Luquet et Kaushik, 1979). II conviendrait cependant d'étendre l'étude physiologique à des protéines autres que la caséine $\alpha_{\mathrm{s}}$ et d'immunoglobuline. Dans cette optique, une étude similaire à la 
présente, mais dont le but serait de retracer l'apparition d'une protéine exogène dans le tissu du segment postérieur, suite à une incubation in vivo ou in vitro, permettrait alors de statuer définitivement sur la capacité de l'intestin de la Truite arc-en-ciel, à prendre en charge des protéines intactes.

Reçu en juin 1983.

Accepté en novembre 1983.

Remerciements. - Ce travail a été rendu possible par des subventions du Conseil des recherches en sciences naturelles et en génie du Canada (CRSNG) et le Fonds FCAC du Québec (à Joël de la Noüe). Les auteurs remercient le Centre de recherche en nutrition pour l'aide accordée à Guy Marcotte.

\section{Références}

BERGOT P., 1976. Démonstration par le rouge de ruthénium d'invaginations profondes de la membrane plasmique apicale des entérocytes dans l'intestin postérieur chez la truite arc-enciel. Ann. Biol. anim. Biochim. Biophys., 16, 37-42.

BERTIN L., 1958. L'appareil digestif, 1268-1273. In GRASSÉ P. P., Traité de zoologie, Agnates et poissons, Masson et Cie, Paris.

BOGÉ G., RIGAL A., PERES G., 1979. A study of energized transport mechanisms of glycine absorption by the rainbow trout (Salmo gairdnerii, Rich.). Comp. Biochem. Physiol., 64A, 537-541.

BRAMBELL F. W. R. 1958. The passive immunity of the young mammal. Biol. Rev., 33, 488-531.

BURNSTOCK G., 1959. The morphology of the gut of the brown trout, Salmo trutta. Quart. J. Microsc. Sci, 100, 183-198.

CRANE R. K., WILSON T. H., 1958. In vitro method for the study of the rate of intestinal absorption of sugars. J. appl. Physiol., 13, 145-146.

CLARK S. L., 1959. The ingestion of proteins and colloidal materials by columnar absorptive cells of the small intestine in suckling rats and mice. J. biophys. biochem. Cytol., 5, 41-50.

EZEASOR D. N., STOKOE W. M., 1980. Scanning electron microscopic study of the gut mucosa of the rainbow trout. J. Fish Biol., 17, 529-539.

EZEASOR D. N., STOKOE W. M., 1981. Light and electron microscopic studies on the absorption cells of the intestine, caeca and rectum of the adult rainbow trout, Salmo gairdnerii (Rich.). J. Fish Biol., 18, 527-544.

HEMMINGS W. H., 1978. Carriage of dietary protein into the tissues. Specul. sci. Technol., 1. 129-134.

HOLMES W. M., SCOTT G. H., 1960. Studies of the respiratory rate of excretory tissues in the cutthroat trout, Salmo clarkii, I and II. Physiol. Zool., 33, 9-20.

HUGON J. S., 1971. Absorption of horseradish peroxidase by the mucosal cells of the duodenum of mouse. II. The newborn mouse. Histochimie, 26, 19-27.

IWAI T., 1968. Fine structure and absorption pattern of intestinal epithelial cells in rainbow trout alevins. Z. Zellforsch. Mikrosk. Anat., 91, 366-379.

JONES R. $E_{\ldots}$ 1976. The selective uptake and transmission of protein to the circulation from the small intestine of the sucking rat. Biochem. biophys. Acta, 451, 151-160.

JOVIN T., CHRAMBACH A., NAUGHTON M. A., 1964. An apparatus for preparative temperatureregulated polyacrylamide gel electrophoresis. Anal. Biochem., 9, 351-369.

KRAEHENBUHL J.-P., CAMPICHE M. A., 1969. Early stages of intestinal absorption of specific antibodies in the newborn - An ultrastructural, cytochemical and immunological study in pig, rat and rabbit. J. Cell Biol., 42, 345-362. 
LIAO P. B., MAYO R. D., 1972. Salmonid hatchery water reuse systems. Aquaculture, 1, 315-335.

LUQUET P., KAUSHIK S. J., 1979. Besoins en protéines et en acides aminés. Actes Coll. C.N.E.R.N.A., Paris, mai, 171-183.

MARCOTTE G., DE LA NOÜE J., 1983. In vitro intestinal absorption of glycine and L-alanine in rainbow Trout, Salmo gairdnerii. Comp. Biochem. Physiol. (sous presse).

MORRIS I. G., 1968. Gamma globulin absorption in the membrane, 1491-1512. In HEIDEL W., Handbook of physiology., Vol. 3, Sect. VI, Am. Physiol. Soc., Washington, D. C.

NOALLIAC-DEPEYRE J., GAS N., 1973. 'Absorption of protein macromolecules by the enterocytes of the carp. Ultrastructural and cytochemical study. Z. Zellforsch, 146, 525-541.

PETTIGREW T., HENDERSON E. B., SAUNDERS R. L., SOCHASKY J. B., 1978. A review of water reconditioning re-use technology for fish culture with a selected bibliography. Fish. Mar. Service Tech., Report 801, IV + $19 \mathrm{p}$.

REISNER A. H., NEMES P., BUCHOLTZ C., 1975. The use of coomassie brillant blue G-250 perchloric acid solution for staining in electrophoresis and isoelectric focussing on polyacrylamide gels. Anal. Biochem., 64, 509-516.

RODEWALD R., 1973. Intestinal transport of antibody in the newborn rat. J. Cell Biol., 58, $189-211$.

SIMARD C., DUPONT Y., BOULET M., 1979. Etude de la dépolymérisation de fractions des protéines de soja, de féverole et de colza. J. Inst. can. Sci. Technol. Aliment., 12, 66-71.

TRIPATHI K. K., GEHRKE C. W., 1970. Chemical and chromatographic isolation of $\kappa$-casein. J. Chromatog., 46, 280-285.

WILLIAMS E. W., HEMMINGS W. A., 1978. Intestinal uptake and transport of proteins in the adult rat. Proc. roy. Soc. Lond B., 203, 177-189.

WILSON T. H., WISEMAN G., 1954. The use of sacs of everted small intestine for study of the transference of substances from the mucosal to the serosal surface. J. Physiol. (London), 123, $116-125$.

YAMAMOTO T., 1966. An electron microscope study of the columnar epithelial cell in the intestine of freshwater teleosts: goldfish (Carassius auratus) and rainbow trout (Salmo irideus). Z. Zellforsch. Microsk. Anat., 72, 66-87. 